CARDIOVASCULAR MEDICINE

\title{
Influence of arterial compliance on presence and extent of ischaemia during stress echocardiography
}

\author{
B A Haluska, K Matthys, R Fathi, E Rozis, S G Carlier, T H Marwick
}

Heart 2006;92:40-43. doi: 10.1136/hrt.2004.052209

See end of article for authors' affiliations

Correspondence to:

Professor Thomas Marwick,

University of Queensland

Department of Medicine,

Princess Alexandra

Hospital, Ipswich Road,

Brisbane, Queensland

4102, Australia;

tmarwick@soms.uq.edu.au

Accepted

10 February 2005

\begin{abstract}
Objective: To seek an association between total arterial compliance (TAC) and the extent of ischaemia at stress echocardiography.

Design: Cohort study.

Setting: Regional cardiac centre.

Methods: 255 consecutive patients (147 men; mean (SD) age 58 (8)) presenting for stress echocardiography for clinical indications were studied. Wall motion score index (WMSI) was calculated and ischaemia was defined by an inducible or worsening wall motion abnormality. Peak WMSI was used to reflect the extent of dysfunction (ischaemia or scar), and $\triangle \mathrm{WMSI}$ was indicative of extent of ischaemia. TAC was assessed at rest by simultaneous radial applanation tonometry and pulsed wave Doppler in all patients.

Results: Ischaemia was identified by stress echocardiography in 65 patients (25\%). TAC was similar in the groups with negative and positive echocardiograms $(1.08 \quad(0.41) \vee 1.17 \quad(0.51) \mathrm{ml} / \mathrm{mm} \mathrm{Hg}$, not significant). However, the extent of dysfunction was associated with TAC independently of age, blood pressure, risk factors, and use of a $\beta$ blocker. Moreover, the extent of ischaemia was determined by TAC, risk factors, and use of a $\beta$ blocker.

Conclusion: While traditional cardiovascular risk factors are strong predictors of ischaemia on stress echocardiography, TAC is an independent predictor of the extent of ischaemia.
\end{abstract}

$\mathrm{T}$ otal arterial compliance (TAC) reflects the properties of the vessel wall and distal vasculature, and is a determinant of arterial pressure and flow. Arterial compliance is reduced with age, vascular disease, and hypertension ${ }^{1-3}$ and is linked with the sequelae of these disorders. Reduction of vessel elasticity leads to an increment of afterload on the heart (as lower arterial compliance is closely related to increased pulse pressure), which in turn leads to left ventricular hypertrophy. ${ }^{4}$ Increased pulse pressure is a determinant of cardiovascular risk and mortality. ${ }^{5-7}$ A stiffer vascular system also leads to lower diastolic pressure, ${ }^{8}$ resulting in a decreased coronary perfusion pressure. ${ }^{9}$

Several studies have shown a correlation between compliance and the presence of significant coronary artery disease (CAD), as might be expected from the association of both abnormal compliance and coronary artery disease with common cardiovascular risk factors. ${ }^{10-12}$ Moreover, arterial compliance may be an important contributor to the provocation of ischaemia at stress testing, and an association has been documented with reduced exercise capacity and the presence of a lower ischaemic threshold..$^{13}{ }^{14}$ We sought to further address the interaction of arterial function and ischaemia by examining the association between compliance and ischaemic burden, reflected by the extent of inducible wall motion abnormality.

\section{METHODS}

\section{Patient selection}

In this prospective, cross sectional study, we studied 255 consecutive patients ( 147 men; mean (SD) age 59 (8) years) of intermediate risk with known or suspected CAD presenting for stress echocardiography for clinical indications: 65 for dobutamine stress echocardiography and 190 for exercise echocardiography. TAC was assessed by simultaneous radial applanation tonometry and pulsed wave Doppler at rest in all patients. Coronary angiograms were recorded at the discretion of the treating cardiologist in 30 patients $(11 \%)$.

\section{Clinical evaluation}

Cardiovascular risk factors that were designated were smoking, hypertension (blood pressure $>140 / 90 \mathrm{~mm} \mathrm{Hg}$ ), diabetes (fasting blood glucose $>6.7 \mathrm{mmol} / \mathrm{l}$ ), hypercholesterolaemia (total cholesterol $>5.2 \mathrm{mmol} / \mathrm{l}$ ), previous myocardial infarction, and previous cardiac intervention. Most patients were receiving one or more of the following medical treatments: $\beta$ blockers, angiotensin converting enzyme inhibitors, calcium antagonists, long acting nitrates, or statins. Medical treatment was discontinued before the stress echocardiography according to the referring physician.

\section{Stress echocardiography}

All patients underwent stress echocardiography, either dobutamine or exercise echocardiography, based on anticipated functional capacity. After patients were prepared for standard 12 lead ECG monitoring and resting blood pressure was obtained, resting echocardiographic images (five views) were digitally acquired as cineloops consisting of one cardiac cycle. For exercise echocardiography, patients underwent a maximal treadmill test under a Bruce protocol. For dobutamine stress echocardiography, dobutamine was infused at a rate of $5,10,20,30$, and $40 \mu \mathrm{g} / \mathrm{kg} / \mathrm{min}$ in three minute stages. Echocardiographic images were obtained at each stage on videotape and digitally acquired at rest, low dose ( 5 or $10 \mu \mathrm{g} /$ $\mathrm{kg} / \mathrm{min}$ ), pre-peak infusion, and peak infusion. Blood pressure, heart rate, ECG changes, and symptoms were also monitored at each stage. Standard end points were used to terminate the test. A diagnostic test was defined as reaching $>85 \%$ maximum predicted heart rate, and patients were

Abbreviations: $C A D$, coronary artery disease; TAC, total arterial compliance; WMSI, wall motion score index 
excluded if they did not reach $>85 \%$ maximum predicted heart rate.

The digital stress echocardiographic images were stored on magneto-optical disk and analysed off line by an expert observer. A 16 segment model was used for wall motion scoring and a positive test was defined by an inducible or worsening wall motion abnormality. Ischaemia was defined by a new or worsening wall motion abnormality. Wall motion score index (WMSI) was calculated by the method previously described. ${ }^{15}$ Peak WMSI was considered to reflect total ischaemic burden (that is, ischaemia or scar) and change in WMSI was used to represent the extent of ischaemia.

\section{Total arterial compliance}

TAC was calculated by the pulse-pressure method by simultaneously acquiring radial applanation tonometry and pulsed wave Doppler and two dimensional echocardiographic measurement of the left ventricular tract. The pulse-pressure method calculates TAC from a combination of central pressure, derived from radial applanation tonometry and the use of a transfer function, and stroke volume, derived from pulsed Doppler of the left ventricular outflow. ${ }^{16}$ Measurements were taken after 5-10 minutes of rest. Firstly, the patient's blood pressure was measured in the left arm with a standard sphygmomanometer and the pressures were entered into the acquisition program. The radial artery was located and all patients had tonometry (Millar SPT-301 Mikro-Tip transducer, Millar Instruments, Houston, Texas, USA) in the left arm. Standard resting echocardiographic images were obtained with the patient in a left lateral decubitus position (Vingmed System 5 with a $1.7 \mathrm{MHz}$ harmonic imaging probe, GE Medical Systems, Milwaukee, Wisconsin, USA). The left ventricular outflow tract diameter was measured off line from the digitally stored parasternal long axis view and entered into the analysis program. When optimal Doppler and tonometry signals were found, three sets of 20-30 cardiac cycles of tonometry and Doppler data (both timed to the ECG) were digitised simultaneously

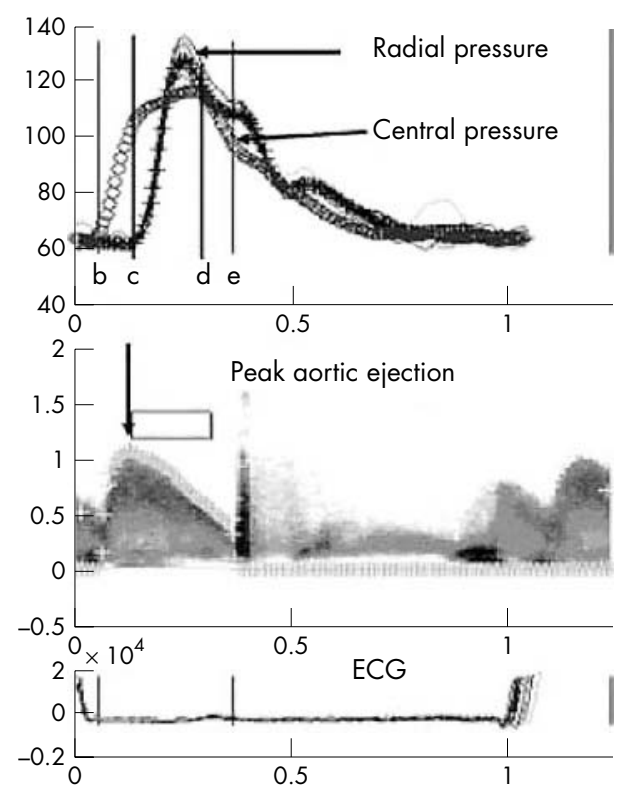

Figure 1 A customised analysis program was used to determine mean pressure and flow values and to calculate a mean aortic pressure waveform by a transfer function model. Radial and derived central pressure wave forms are shown with arrows. Cursors are used to identify beginning aortic ejection (b), peak aortic ejection (c), peak pulse pressure (d), and end aortic ejection (e).
(WaveBook 512, IOTech Inc, Cleveland, Ohio, USA), integrated by specialised software, and transferred to a laptop computer. Data were stored digitally for offline analysis by a custom analysis program written in MATLAB 4 (The MathWorks, Natick, Massachusetts, USA).

Specific cardiac cycles of tonometry and Doppler were chosen from the raw dataset based on data quality and averaged for analysis; depending on data quality this amounted to 4-15 cycles. After identifying the Doppler signal, beginning and end of ejection, peak ejection, and peak pulse pressure on both Doppler and pressure signals, the analysis program determined mean pressure and flow values and calculated a mean aortic pressure based on the transfer function (fig 1). ${ }^{16}$ Mathematical algorithms in the analysis program are used to derive values for compliance and other haemodynamic indices from the pressure and flow data.

\section{Statistical analysis}

Statistical analysis was undertaken by commercially available software (SPSS version 9.0; SPSS Inc, Chicago, Illinois, USA). Continuous data were presented as mean (SD). Independent samples $t$ test was performed to compare the positive with the negative stress echocardiography group. Linear regression was performed, together with univariate and multivariate models, to determine predictors of peak WMSI (ischaemia) and $\triangle$ WMSI (extent of ischaemia).

\section{RESULTS}

\section{Clinical characteristics}

Table 1 summarises the clinical features of the study group. They conform to a typical group undergoing stress echocardiography for known or suspected coronary disease. Although the dobutamine and exercise echocardiography groups differed in age (62 (10) $v 57$ (7) years, $\mathrm{p}<0.0001)$ and the prevalence of diabetes mellitus (23\% $v 52 \%$, $\mathrm{p}<0.003)$, the prevalence of hypertension, overall numbers of risk factors, and treatment with angiotensin converting enzyme inhibitors and statins were similar. Importantly, systolic blood pressure (129 (27) v 132 (19) mm Hg, not significant) and pulse pressure (58 (21) v 53 (16) $\mathrm{mm} \mathrm{Hg}$, not significant) were comparable, as was TAC ( $1.17(0.510 \mathrm{v}$ 1.08 (0.41), not significant).

This test was positive for ischaemia in 65 patients $(25 \%)$. Table 2 compares the clinical and haemodynamic features of the patient group with a negative versus the group with a positive stress echocardiogram. As might be expected, patients with a positive scan were older, more of them were men, and they were more likely to be taking antianginal drugs. Although the group with positive stress echocardiography also had a higher prevalence of smoking and diabetes, of the cardiovascular risk factors, only hypertension was

\begin{tabular}{|ll|}
\hline $\begin{array}{l}\text { Table } 1 \\
(\mathrm{n}=255)\end{array}$ & Patient clinical characteristics \\
\hline Age (years) & $58(8)$ \\
Men & $147(57 \%)$ \\
Smoker & $92(36 \%)$ \\
Hypertension & $164(64 \%)$ \\
Diabetes mellitus & $113(44 \%)$ \\
Hypercholesterolaemia & $118(46 \%)$ \\
$\beta$ Blocker & $57(22 \%)$ \\
ACE inhibitor & $82(32 \%)$ \\
Calcium antagonist & $80(31 \%)$ \\
Statin & $83(32 \%)$ \\
Number of risk factors & $2(1)$ \\
Number of medications & $1(1)$ \\
\hline Data are mean (SD) or number $(\%)$. \\
ACE, angiotensin converting enzyme. \\
\hline
\end{tabular}


Table 2 Clinical characteristics, blood pressure, and compliance for patients with positive and negative stress echocardiography results

\begin{tabular}{|c|c|c|c|}
\hline & \multicolumn{2}{|c|}{$\begin{array}{l}\text { Stress echocardiography } \\
\text { result }\end{array}$} & \multirow[b]{2}{*}{ p Value } \\
\hline & Positive & Negative & \\
\hline Number & 65 & 190 & \\
\hline Age (years) & $62(10)$ & $57(7)$ & $<0.0001$ \\
\hline Men & $47(72 \%)$ & $100(52 \%)$ & $<0.005$ \\
\hline Smoker & $38(58 \%)$ & $56(29 \%)$ & $<0.0001$ \\
\hline Hypertension & $39(60 \%)$ & $127(66 \%)$ & NS \\
\hline Diabetes & 15 (23\%) & $99(52 \%)$ & $<0.003$ \\
\hline Increased cholesterol & 35 (54\%) & $83(43 \%)$ & $<0.01$ \\
\hline$\beta$ Blocker & $31(47 \%)$ & 27 (14\%) & $<0.0001$ \\
\hline ACE inhibitor & $18(27 \%)$ & $65(34 \%)$ & NS \\
\hline Calcium antagonist & $24(37 \%)$ & $57(30 \%)$ & $<0.01$ \\
\hline Statin & $24(37 \%)$ & $60(31 \%)$ & NS \\
\hline Number of risk factors & $2(1)$ & $2(1)$ & NS \\
\hline Number of medications & $2(1)$ & $1(1)$ & $<0.0001$ \\
\hline $\mathrm{SBP}(\mathrm{mm} \mathrm{Hg})$ & $129(27)$ & $132(19)$ & NS \\
\hline $\mathrm{DBP}(\mathrm{mm} \mathrm{Hg})$ & $71(11)$ & $78(10)$ & $<0.0001$ \\
\hline $\mathrm{MAP}(\mathrm{mm} \mathrm{Hg})$ & 90 (15) & 96 (13) & $<0.003$ \\
\hline $\mathrm{PP}(\mathrm{mm} \mathrm{Hg})$ & $58(21)$ & $53(16)$ & NS \\
\hline $\mathrm{CO}(\mathrm{l} / \mathrm{min})$ & $5.2(1.5)$ & $4.7(1.2)$ & $<0.007$ \\
\hline TVR $\left(\mathrm{mm} \mathrm{Hg} / \mathrm{ml}^{*} \mathrm{~s}^{-1}\right)$ & $1.1(0.38)$ & $1.3(0.39)$ & $<0.001$ \\
\hline $\mathrm{TAC}(\mathrm{ml} / \mathrm{mm} \mathrm{Hg})$ & $1.17(0.51)$ & $1.08(0.41)$ & NS \\
\hline
\end{tabular}

$\mathrm{CO}$, cardiac output; DBP, diastolic blood pressure; $M A P$, mean arterial pressure; NS, not significant; $\mathrm{PP}$, pulse pressure; SBP, systolic blood pressure; TAC, total arterial compliance; TVR, total vascular resistance.

correlated with TAC $(r=-0.20, \mathrm{p}<0.01)$. Figure 2 summarises the relation between compliance and pulse pressure in the negative and positive echocardiography groups.

\section{Association between extent of ischaemia and compliance}

Of the patients with a positive stress echocardiography, 44 (67\%) had ischaemia (with or without scar) and 19 (29\%) had scar only. In the group with positive stress echocardiograms, WMSI was $1.5(0.5)$ at rest, peak WMSI was $1.6(0.5)$, and $\triangle$ WMSI was $0.1(0.4)$. In a subgroup of $30(46 \%)$ patients with a positive stress echocardiography who underwent cardiac catheterisation, the angiography results gave a mean jeopardy score of 4.6 (3.6). There was no significant correlation between jeopardy score and WMSI at rest, peak WMSI, or $\triangle$ WMSI. There was, however, a correlation between peak WMSI and pulse pressure $(r=0.11$, $\mathrm{p}=0.05)$, as well as mean aortic pressure $(r=-0.14$, $\mathrm{p}=0.01)$.

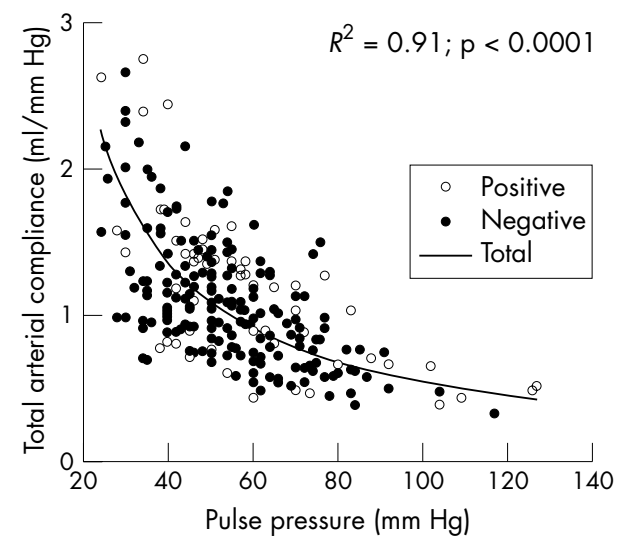

Figure 2 Relation of compliance to pulse pressure in patients with negative and positive stress echocardiography results.
Table 3 Correlates of peak wall motion score index (indicating the presence of coronary artery disease)

\begin{tabular}{|c|c|c|c|c|c|}
\hline \multicolumn{3}{|c|}{ Univariate analysis } & \multicolumn{3}{|c|}{ Multivariate analysis } \\
\hline Variable & $R^{2}$ & p Value & Variable & $R^{2}$ & $p$ Value \\
\hline Age & 0.229 & 0.009 & NA & NA & NA \\
\hline SBP & 0.247 & 0.002 & NA & NA & NA \\
\hline DBP & 0.270 & $<0.0001$ & NA & NA & NA \\
\hline MAP & 0.224 & 0.009 & NA & NA & NA \\
\hline $\mathrm{CO}$ & 0.210 & 0.02 & NA & NA & NA \\
\hline TAC & 0.221 & 0.01 & TAC & 0.152 & 0.005 \\
\hline Smoking & 0.362 & $<0.0001$ & Smoking & 0.338 & $<0.0001$ \\
\hline$\beta$ Blocker & 0.323 & $<0.0001$ & $\beta$ Blocker & 0.219 & $<0.0001$ \\
\hline
\end{tabular}

Multiple linear regression models were constructed to determine univariate and multivariate predictors for peak WMSI (total extent of abnormal function) and $\Delta$ WMSI (extent of ischaemia). Tables 3 and 4 show the significant associations. Variables entered into the model were cardiovascular risk factors, medications, and pressure and flow characteristics. Compliance was shown in these analyses to be independently associated with both peak WMS and $\Delta$ WMSI, even after accounting for age, blood pressure, risk factors, and $\beta$ blocker use.

\section{DISCUSSION}

This study confirms the association of reduced arterial compliance with more extensive ischaemia. These findings reinforce the importance of vascular function not only to cardiac function but also to the results of stress echocardiography. The lack of a significant difference in TAC between the groups with negative and positive stress echocardiography results probably reflects the high incidence of risk factors and medications in both groups.

\section{Compliance as a determinant of CAD}

Aortic stiffness and left ventricular mass are increased in patients with CAD. ${ }^{10}$ However, the understanding of how these entities interact is still limited. As wave reflections are stronger in a stiffer vascular system, resulting in a higher augmentation index, it has been shown that increased augmentation index, and hence decreased compliance, is associated with an increased risk of CAD. ${ }^{17} \mathrm{TAC}$ is probably a predictor of CAD, but not a strong one, especially when traditional risk factors are also accounted for (as shown in the univariate and multivariate linear regression models), as demonstrated in previous studies with the presence of significant CAD. ${ }^{11}{ }^{12}$ The role of compliance in ischaemia needs to take into account the blood pressure of these patients-in fact, the similarity of TAC between the groups in the face of lower blood pressure in the patients with a

Table 4 Correlates of $\Delta$ wall motion score index (indicating extent of coronary artery disease)

\begin{tabular}{|c|c|c|c|c|c|}
\hline \multicolumn{3}{|c|}{ Univariate } & \multicolumn{3}{|c|}{ Multivariate } \\
\hline Variable & $R^{2}$ & p Value & Variable & $R^{2}$ & p Value \\
\hline Age & 0.161 & 0.02 & NA & NA & NA \\
\hline SBP & 0.193 & 0.002 & NA & NA & NA \\
\hline MAP & 0.147 & 0.05 & NA & NA & NA \\
\hline $\mathrm{CO}$ & 0.202 & 0.001 & NA & NA & NA \\
\hline TAC & 0.184 & 0.003 & TAC & 0.164 & 0.002 \\
\hline Smoking & 0.19 & $<0.0001$ & Smoking & 0.314 & $<0.0001$ \\
\hline$\beta$ Blocker & 0.232 & $<0.0001$ & $\beta$ Blocker & 0.200 & $<0.0001$ \\
\hline
\end{tabular}


positive stress echocardiogram probably reflects the effects of treatment.

\section{Interaction of arterial compliance with ischaemia}

The extent of ischaemia reflects an interplay between supply and demand, and both are influenced by compliance. On the one hand, ejecting into a stiffer systemic tree increases cardiac load and myocardial oxygen consumption (increased demand). ${ }^{18}$ On the other hand, a loss of compliance induces an increase in pulse pressure, resulting from an increase in systolic and a decrease in diastolic pressure. ${ }^{8}$ The systolic pressure increase contributes to the increased cardiac load, ${ }^{18}$ whereas the decrease in diastolic pressure reduces coronary perfusion (decreased supply). ${ }^{9}$ Previous work has shown an association of compliance with exercise capacity and ischaemic threshold. ${ }^{13}{ }^{14}$ In healthy people, exercise capacity is related to maximum cardiac output, which may be influenced by systemic compliance. ${ }^{13}$ Indeed, Cameron et al ${ }^{19} 20$ already reported a correlation between lower arterial stiffness and higher levels of aerobic fitness.

However, in patients with CAD exercise capacity is related to myocardial ischaemia, the threshold of which is mainly determined by coronary perfusion and cardiac workload; thus, the interaction between supply and demand. A stiffer systemic vasculature may lower ischaemic threshold. ${ }^{14}$ Kingwell et al $^{14}$ did not assess myocardial ischaemia with an imaging technique, as was done in the present study, but by means of evaluating the ST segment depression on the ECG during a treadmill test. Those investigators found that various measures of arterial stiffness (arterial compliance, pulse wave velocity, and augmentation index) inversely correlated with time to ischaemia, independent of potential confounding factors such as sex, age, or mean arterial pressure. Our data supplement these findings by showing that TAC was a predictor not only of peak WMS (ischaemia and resting dysfunction) but also of $\triangle$ WMS (extent of ischaemia).

\section{Clinical implications}

While traditional cardiovascular risk factors are strong predictors of ischaemia on stress echocardiography, reduction of TAC indicates the extent of ischaemia on stress echocardiography. Some clinical consequences may be anticipated: interventions such as smoking cessation, aggressive lipid lowering, hypertension control, and exercise may have a favourable impact on TAC and therefore improve ischaemia. Other associations, including the detection of ischaemia at lower workload or with less severe stenoses, and even the development of false positive scan results, warrant investigation in other cohorts.

\section{Authors' affiliations}

B A Haluska, K Matthys, R Fathi, E Rozis, T H Marwick, University of Queensland, Brisbane, Australia

S G Carlier, Cardiovascular Research Foundation, New York, New York, USA

\section{REFERENCES}

1 Safar ME, London GM. Arterial and venous compliance in sustained essential hypertension. Hypertension 1987;10:133-9.

2 London GM, Pannier B, Guerin AP, et al. Cardiac hypertrophy, aortic compliance, peripheral resistance, and wave reflection in end-stage renal disease: comparative effects of ACE inhibition and calcium channel blockade. Circulation 1994;90:2786-96.

3 McVeigh GE, Bratteli CW, Morgan DJ, et al. Age-related abnormalities in arterial compliance identified by pressure pulse contour analysis: aging and arterial compliance. Hypertension 1999;33:1392-8.

4 Rajkumar C, Cameron JD, Christophidis N, et al. Reduced systemic arterial compliance is associated with left ventricular hypertrophy and diastolic dysfunction in older people. J Am Geriatr Soc 1997:45:803-8.

5 Benetos A, Safar M, Rudnichi A, et al. Pulse pressure: a predictor of long-term cardiovascular mortality in a French male population. Hypertension 1997;30:1410-5

6 Franklin SS, Khan SA, Wong ND, et al. Is pulse pressure useful in predicting risk for coronary heart Disease? The Framingham heart study. Circulation 1999; 100:354-60.

7 Kannel WB, Vasan RS, Levy D. Is the relation of systolic blood pressure to risk of cardiovascular disease continuous and graded, or are there critical values? Hypertension 2003:42:453-6.

8 Randall OS, van den Bos GC, Westerhof N. Systemic compliance: does it play a role in the genesis of essential hypertension? Cardiovasc Res 1984; 18:455-62

9 Kass DA, Saeki A, Tunin RS, et al. Adverse influence of systemic vascular stiffening on cardiac dysfunction and adaptation to acute coronary occlusion. Circulation 1996:93:1533-41.

10 Gatzka CD, Cameron JD, Kingwell BA, et al. Relation between coronary artery disease, aortic stiffness, and left ventricular structure in a population sample. Hypertension 1998;32:575-8.

11 Herrington DM, Kesler K, Reiber JHC, et al. Arterial compliance adds to conventional risk factors for prediction of angiographic coronary artery disease. Am Heart J 2003;146:662-7.

12 Syeda B, Gottsauner-Wolf M, Denk S, et al. Arterial compliance: a diagnostic marker for atherosclerotic plaque burden? Am J Hypertens 2003;16:356-62.

13 Kingwell BA. Large artery stiffness: implications for exercise capacity and cardiovascular risk. Clin Exp Pharmacol Physiol 2002;29:214-7.

14 Kingwell BA, Waddell TK, Medley TL, et al. Large artery stiffness predicts ischaemic threshold in patients with coronary artery disease. J Am Coll Cardiol 2002;40:773-9.

15 Olmos LI, Dakik H, Gordon R, et al. Long-term prognostic value of exercise echocardiography compared with exercise 201Tl, ECG, and clinical variables in patients evaluated for coronary artery disease. Circulation 1998;98:2679-86.

16 Chen $\mathrm{CH}$, Nevo E, Fetics B, et al. Estimation of central aortic pressure waveform by mathematical transformation of radial tonometry pressure: validation of generalized transfer function. Circulation 1997;95:1827-36.

17 Hayashi T, Nakayama Y, Tsumura K, et al. Reflection in the arterial system and the risk of coronary heart disease. Am J Hypertens 2002;15:405-9.

18 Kelly RP, Tunin R, Kass DA. Effect of reduced aortic compliance on cardiac efficiency and contractile function of in situ canine left ventricle. Circ Res 1992;71:490-502.

19 Cameron JD, Dart AM. Exercise training increases total systemic arterial compliance in humans. Am J Physiol 1994;266:H693-701.

20 Cameron JD, Rajkumar C, Kingwell BA, et al. Higher systemic arterial compliance is associated with greater exercise time and lower blood pressure in a young older population. J Am Geriatr Soc 1999;47:653-6.

\section{1 th European Forum on Quality Improvement in Health Care}

26-28 April 2006, Prague, Czech Republic For further information please go to: www.quality.bmipg.com Book early to benefit from a discounted delegate rate 